\title{
Dental pain induced by an ambient thermal differential: pathophysiological hypothesis
}

This article was published in the following Dove Press journal: Journal of Pain Research

\author{
Anaïs Le Fur-Bonnabesse ${ }^{1,2}$ \\ Céline Bodérél-3 \\ Cyrielle Hélou ${ }^{2}$ \\ Valérie Chevalier ${ }^{2,4}$ \\ Jean-Paul Goulet ${ }^{5}$ \\ 'Laboratory of Neurosciences of \\ Brest (EA4685), University of Western \\ Brittany, Brest, France; ' 2 Dental School, \\ University of Western Brittany, \\ Brest, France; ${ }^{3}$ Assessment and \\ Treatment Center of Pain, Regional \\ and University Hospital Center, Brest, \\ France; ${ }^{4}$ Laboratory IRDL, FRE CNRS \\ 3744, University of Western Brittany, \\ Brest, France; ${ }^{5}$ School of Dental \\ Medicine, Universite Laval, Quebec, \\ QC, Canada
}

\begin{abstract}
Dental pain triggered by temperature differential is a misrecognized condition and a form of dental allodynia. Dental allodynia is characterized by recurrent episodes of diffuse, dull and throbbing tooth pain that develops when returning to an indoor room temperature after being exposed for a long period to cold weather. The pain episode may last up to few hours before subsiding. Effective treatment is to properly shield the pulpal tissue of the offending tooth by increasing the protective layer of the dentin/enamel complex. This review underscores the difference in dentin hypersensitivity and offers a mechanistic hypothesis based on the following processes. Repeated exposure to significant positive temperature gradients (from cold to warm) generates phenotypic changes of dental primary afferents on selected teeth with subsequent development of a "low-grade" neurogenic inflammation. As a result, nociceptive C-fibers become sensitized and responsive to innocuous temperature gradients because the activation threshold of specific TRP ion channels is lowered and central sensitization takes place. Comprehensive overviews that cover dental innervation and sensory modalities, thermodynamics of tooth structure, mechanisms of dental nociception and the thermal pain are also provided.
\end{abstract}

Keywords: pain, dental pain, thermal allodynia, atypical tooth sensitivity, dentin hypersensitivity

\section{Introduction}

The mechanisms underlying dentin hypersensitivity (DH) evoked by thermal stimuli are still poorly understood even if dental pain is probably one of the most common acute pain complaints.

Typical DH is a sudden short, sharp pain of rapid onset following an appropriate stimulus that activates nociceptors in the pulp/dentin border area. Although cold is the most frequently reported offending trigger, the pain can be evoked by hot as well as evaporative, tactile, osmotic and chemical stimuli. The consensus around the case definition of DH highlights the increased stimulus transmission because of exposed dentin and opening of the dentinal tubule network. ${ }^{1,2}$ The diagnosis of DH means that the pain cannot be explained as arising from any other dental defect or pathology.

Unlike typical $\mathrm{DH}$, this review focuses on atypical dental sensitivity (thermal allodynia) in patients experiencing thermal differential between outdoors and indoors. Symptoms occur in autumn or winter, when outside temperatures are cold. The temperature gradient between outside and inside (the thermal differential) is important. It is not a direct response to thermal stimulation caused by a typical $\mathrm{DH}$, but it is the thermal differential that causes pain. The quality of dental pain is different from that
Correspondence: Anaïs Le FurBonnabesse

Faculté d'odontologie, Université de Bretagne Occidentale, 22, Avenue

Camille Desmoulins - CS93837, 29200

Brest, France

Tel +332980I 6489

Fax +33298016932

Email anais.lefurbonnabesse@univ-brest.fr 
involved in typical DH: pain is diffuse, dull and throbbing. It appears after a while and can last for over an hour.

The aims of this article was to report an unusual type of dental hypersensitivity and present potential pathophysiological processes leading to delayed dental pain caused by ambient thermal differential.

\section{Clinical context}

In recent years patients have been referred to the dental school in Quebec city for tooth pain occurring at room temperature after being outdoors in a cold environment. They had seen their dentist on several occasions because of remittent dental pain of unknown cause. Treatments for DH (desensitizing toothpaste, Duraphat $^{\circledR}$, Gluma ${ }^{\circledR}$, etc.) were not successful. The characteristics and symptoms of three patients are listed in Table 1.

The symptoms occurred during the autumn and winter seasons when the outside temperature was cold enough. Patients were reporting a poorly localized, dull and throbbing pain that started within 15 minutes after returning inside following an outdoor activity. Within 5-10 minutes, the pain had reached a peak and then lasted for a half an hour up to 3 hours before it gradually reduced completely. Typically, the pain was moderately severe, and the intensity could reach 8 out of 10 on visual analog scale (VAS). None of the patients was able to precisely pinpoint an offending tooth. The pain could cross the midline and sometimes spread to the opposite dental arch. Paracetamol or ibuprofen helped alleviate the pain somewhat. Moreover, no dental pain was reported, while patients were outside and exposed to a cold temperature before returning indoor.

None of these patients reported a history of tooth hypersensitivity to cold or hot beverages or foods. None of the patients was a mouth breather, and all had noncontributory medical and drug intake status. Upon clinical examination, no pain was evoked with air blast or by scratching the surface of the teeth with a dental probe at the cervical margin in the affected dental arch segment. Pulp reactivity to cold test stimulus with coolant spray (Endo-Ice ${ }^{\odot}$ ) was also normal. The patients' complaint could be reproduced when they were asked to keep ice-cold water in contact with their teeth for at least 30 seconds and then take and retain hot water in their mouth for at least 1 minute. Although the elicited pain was less severe and of a short duration, all the patients could confirm the similarity and familiarity of the evoked pain. Electrical pulp testing of the teeth in the affected dental arch showed at least one tooth with extremely low threshold for tingling/pain compared to the contralateral side.

Applying orthodontic wax, which serves as insulation on the targeted teeth (causal teeth), during outdoor activities, helps to relieve pain. This confirms the diagnosis of pain induced by a thermal differential.

\section{Dental primary afferent neurons and tooth sensory modalities}

Tooth sensitivity rests on primary afferent nerves that have their cell bodies in the trigeminal ganglia (TRG). Sensory afferents entering into the tooth are large-diameter myelinated $\mathrm{A} \beta$-fibers, medium-diameter lightly myelinated $\mathrm{A} \delta$-fibers and small afferent unmyelinated C-fibers. ${ }^{3}$ The $A \delta$-fibers are fastconducting afferents responsible for the instant sharp pain induced by stimuli in the noxious range. The unmyelinated C-fibers are high-threshold nociceptors with slow conduction velocities implicated in the dull aching sensation that develops after the sharp pain. For the most part, C-fiber

Table I Characteristics and symptoms of three patients with atypical tooth sensitivity

\begin{tabular}{|c|c|c|c|}
\hline Characteristics & Patient I & Patient 2 & Patient 3 \\
\hline Gender/age (years) & Female/53 & Female/55 & Female/49 \\
\hline Chief complaint & \multicolumn{3}{|c|}{ Dental pain after being outdoor in a cold environment and going back to a "physiological temperature" environment } \\
\hline \multirow[t]{2}{*}{ Pain characteristics } & Diffuse, dull and throbbing & & \\
\hline & Set off after a short latency and may last several hours & & \\
\hline VAS & 6 & 8 & 8 \\
\hline Pain alleviation & Paracetamol & Paracetamol or ibuprofen & Paracetamol \\
\hline Causal teeth* & ||$-2 \mid$ & $|I-2|-42$ & ||$-2 \mid$ \\
\hline $\begin{array}{l}\text { Clinical examination } \\
\text { findings }\end{array}$ & $\begin{array}{l}\text { Cervical light-cure glaze on exposed dentin at the cervical } \\
\text { margin }(\leq \mathrm{I} \mathrm{mm}) \text {; Class III composite } \mathrm{II}_{M} ; 2 \mathrm{I}_{M}\end{array}$ & $\begin{array}{l}\leq \mathrm{Imm} \text { exposed dentin at } \\
\text { the cervical margin }\end{array}$ & Composite facet \\
\hline Cold test & Normal & Normal & Normal \\
\hline Electrical pulp test & ++ & ++ & + \\
\hline Diagnosis & Dental pain induced by thermal differential allodynia & & \\
\hline Treatment & Veneer facet & Protective soft mouth guard & Veneer facet reflection \\
\hline
\end{tabular}

Note: *Universal tooth numbering system.

Abbreviation: VAS, visual analog scale. 
terminals are found in the pulp itself and respond only to intense stimuli reaching the pulp. Among other small nerve fibers found in the pulp are postganglionic sympathetic efferent nerves that innervate the pulpal blood vessels and parasympathetic nerves possibly implicated in the regulation of pulpal blood flow (PBF).

The major players mediating tooth pain sensations are clearly $\mathrm{A} \delta$-fibers and $\mathrm{C}$-fibers, but a few $\mathrm{A} \beta$-fibers might also act as nociceptors although most are probably low-threshold mechanotransducers. ${ }^{4}$

\section{Tooth thermomechanical behavior}

According to Lin et al, ${ }^{5}$ the thermal tooth behavior relates to both a conduction process and tooth physiological processes. The transfer of heat or cold between the different parts of the tooth and its consequence depend on the thermophysical properties of enamel and dentin along with the physiological processes happening in the dentinal tubule and the pulpal tissue. ${ }^{5}$ The thermal insulating capacity of the enamel prevents hot or cold stimulation eliciting dental pain in an uninflamed healthy tooth; however, thermal variations will affect both the dental structure and the dentinal fluid flow. ${ }^{6}$ Applying a thermal stimulus that creates an initial large temperature gradient at the outermost enamel surface causes rapid flexural deformation before any temperature is transferred deeper into the tooth structure and change is detected at the dentinoenamel junction (DEJ). ${ }^{7}$ Thus, thermally induced enamel flexure could explain the structural deformation observed at the pulpal wall following intense thermal stimulation. ${ }^{7}$ With finite element simulation and thermal stimuli recorded experimentally, Linsuwanont et $\mathrm{al}^{7}$ showed that a flexure (expansion with hot or contraction with cold) follows large temperature gradients at the enamel surface, which then leads almost immediately to a mechanically induced biphasic strain response at the pulpal dentin surface. It is now suggested that this mechanically induced strain response at the pulpal dentin wall initiates the fluid flow movement in the dentinal tubule after thermal stimulation. Thermal stimulation causes dentinal fluid movement. The predominant pattern of fluid flow in the dentinal tubules is inward toward the pulp with heat stimuli and in the opposite direction away from the pulp with cold stimuli. The time required to detect fluid movement in response to thermal stimulation was shorter for hot compared to cold, and for both types of stimuli, initial fluid movement within the dentinal tubule preceded the temperature change at the DEJ by about half a second. ${ }^{6}$ With extreme thermal stimuli, a bidirectional fluid flow has also been observed, that is an initial flow occurring in the opposite direction of the predominant pattern right after the application of the thermal stimulus and before the temperature change is detected at the DEJ. ${ }^{8-11}$

The bidirectional response was also observed more frequently after increasing intrapulpal pressure. Linsuwanont et $\mathrm{al}^{6}$ suggested that, in a clinical situation, the dentinal fluid movement in teeth with pulpal inflammation (increase in intrapulpal pressure) may respond differently to thermal stimuli in comparison with teeth with normal physiological pulpal pressure.

\section{Mechanisms of dental nociception}

Dental pain can result from mechanical, thermal, chemical, evaporative and osmotic stimuli. The exact mode of transmission of stimuli across the tooth structure and more specifically how heat and cold generate the pulpal nerve impulse to elicit a painful sensation are far from clear and the subject of much debate. Three theories for pulp nerve activation have been proposed, but none can entirely explain the various tooth sensory modalities. ${ }^{5}$

"The neural theory" contends that the pulpal nerve endings are directly activated by external stimuli, and the transduction of a specific stimulus to an electrical nerve impulse is mediated by nociceptive receptors expressed in dental primary afferent neurons. Consistent with the most common view, immunohistochemical analysis showed that the majority of dental afferent neurons were small-diameter nociceptors and expressed nociceptive-specific ion channels among which TRPV1, TRPV2, TRPA1, TRPM8, P2X and $\mathrm{P} 2 \mathrm{X}_{3}$ were found in subsets of neurons. ${ }^{12,13}$ TRPA1 and TRPM8 are cold/mechanosensitive and cold-sensitive ion channel receptors with the threshold of $<25^{\circ} \mathrm{C}$ and $<17^{\circ} \mathrm{C}$, respectively, whereas TRPV1 and TRPV2 are warmsensitive receptors with respective thresholds of $>43^{\circ} \mathrm{C}$ and $>52^{\circ} \mathrm{C}$, respectively. ${ }^{14}$ Besides heat, TPRV1 receptors can be activated by capsaicin and at room temperature when the proton concentration is increased $(<\mathrm{pH} 6.0)$ as is frequently seen in inflammatory conditions. ${ }^{15,16}$ Because TRPV1 was shown to induce the release of inflammatory mediators (SP, CGRP), it may play an important role in pain detection and tissue inflammation. TRPA1 is frequently co-expressed with TRPV1, and both are likely to play an integral role in pain and neurogenic inflammation via the activation of sensory nerves at central and peripheral levels. ${ }^{15} \mathrm{P} 2 \mathrm{X}_{2}$ and $\mathrm{P} 2 \mathrm{X}_{3}$ are specific purinergic ionotropic membrane receptors that are activated by extracellular ATP, which has also been shown to potentiate TRPV1 responses by lowering the temperature threshold for heat activation. ${ }^{15}$ 
"The odontoblastic transduction theory" states that the odontoblasts act as sensory transducers of noxious stimuli into electrical signals transmitted to neighboring nerve endings. The strategic location and proximity of odontoblasts with nerve endings along with the various plasma membrane proteins they express make them a key multimodal cell for sensing both external dental stimuli and pulpal changes. ${ }^{17}$ Odontoblasts are ciliated cells lying at the peripheral border of the pulp close to the dentin. They possess a cellular process that extends into the dentinal tubules to approximately one-third of dentin thickness. Primary afferents innervating the dental pulp form a dense neural plexus just beneath the odontoblast layer. Several mechano/thermosensitive channels belonging to the TRP superfamily (TRPV1, TRPV2, TRPV4, TRPA1, TRPM8) are expressed on the odontoblastic membrane..$^{8,14,17-21}$ These receptors may enable odontoblasts to detect fluid movements within the dentinal tubules that are induced by external stimuli and thus play a role in the transduction of heat and cold stimuli as well as dental pain. El Karim et $\mathrm{al}^{14}$ have clearly demonstrated a potential role for TRPA1 and TRPM8 in transduction of noxious cold responses in odontoblasts as well as TRPV1 responses to noxious heat. Odontoblasts may have a critical role not only in transduction of thermal stimuli but also in mediating pain and inflammation. The direct excitability of odontoblasts is now regarded as highly plausible with the identification of voltage-gated (NA, K) and calcium-activated channels. ${ }^{13,17}$ Shibukawa et $\mathrm{al}^{20}$ provided good evidence that odontoblasts could indeed act as sensory cells and detect the hydrodynamic force inside dentinal tubules. These authors showed that mechanically stimulated odontoblasts release ATP through PANX-1 that can act as a neurotransmitter for $\mathrm{P} 2 \mathrm{X}_{3}$ receptors located on pulpal neurons to drive the sensory transduction sequence for eliciting dental pain. ${ }^{20}$ This has led them to propose a new model called "odontoblast hydrodynamic receptor theory" to explain the sensory generation mechanism underlying dentinal pain. ${ }^{20}$

"The hydrodynamic theory" is the most widely accepted theory which involves dentin sensitivity on stimuli-induced fluid movements in the dentinal tubules and the consequent activation of nociceptors in the pulp/dentin border area. , $8,10,11$ Because dentinal fluid is a natural hydraulic linkage between the environment and the pulp nerve endings, flow changes would explain tooth pain elicited by innocuous stimuli such as an air puff or water spray on dentin lacking enamel coverage. Mechanical forces generated by the movement of dentinal fluid can create sufficient shear stresses on intratubular nerve terminals to activate putative mechanosensitive ion channels and induce dental pain sensation..$^{9,10}$ This is in line with data showing that fluid movement within dentinal tubules in response to thermal stimulation precedes temperature change at the DEJ and pulpal surface of the dentin. 5,6,9 As mentioned earlier, the predominant dentinal fluid flow is outward with cold stimulation and inward with hot stimulation. The velocity of fluid movement occurring between the tubular wall and the odontoblast cellular process extending into the tubules with beaded nerve terminals may produce enough shear forces to activate ion channels colocated on odontoblast and nerve endings. These activations generate nerve impulses and cause sensory response. ${ }^{6}$

\section{Additional considerations Pulpal blood flow and thermoregulation}

Thermoregulation is controlled by vascular responses under the influence of the autonomic nervous system. ${ }^{22}$ Tissue cooling causes fast arteriolar and capillary vasoconstriction, while vasodilation will counteract the warming of tissue. Raynaud's phenomenon is a common clinical disorder with symptoms often associated with exposure to cold temperatures. ${ }^{23}$ During the recovery stage, the vasodilatation due to tissue warming causes pain. Although Raynaud's phenomenon continues to elude investigators, several central and peripheral mechanisms are believed to be involved in the underlying pathophysiological processes (vascular and intravascular abnormalities, endothelial dysfunction, neural abnormalities). ${ }^{23}$ The pulp is a highly vascularized tissue, and the PBF has a significant role in tooth thermal behavior, that is, a significant increase when the temperature rises above $42^{\circ} \mathrm{C}$ and a significant decrease during cooling. ${ }^{5,24}$ It is then possible that the vascular thermoregulation of pulpal tissue following a sustained exposure to a cold outdoor temperature increases the intrapulpal pressure when returning to a warmer temperature, which in turn activates ion channel thermo/mechanosensitive receptors co-localized on nerve endings and odontoblasts. The magnitude of the thermoregulatory process that such temperature change could elicit and how it would affect PBF have yet to be investigated. ${ }^{7}$ On the other hand, the vasoactive neuropeptides SP and CGRP released by $\mathrm{C}$-fiber activation following temperature stimuli may increase PBF rate and thus the intrapulpal pressure. ${ }^{24}$ However, some authors consider the influence of PBF negligible in clinical settings because the pulpal vasculature blood volume is too low. ${ }^{5}$

\section{Bleaching sensitivity}

Patients undergoing teeth-bleaching procedures often complain of unprovoked painful or tingling sensations for a period of time. ${ }^{25}$ The main mechanism underlying bleaching pain is 
not intradental activation of mechanosensitive ion channels by fluid shift in the dentinal tubules (hydrodynamic theory). With their low molecular weight and capacity to denature tissue protein, bleaching agents diffuse through enamel and into the dentin. They contain free radicals and release toxic components that have the capability under certain circumstances to reach the dental pulp and thus induce an inflammatory response especially when dentin thickness fails to provide a good enough protective barrier. ${ }^{26}$ The presence of free radicals generates oxidation by-products shown to be potent activators of cold sensor TRPA1 channels expressed on $\mathrm{C}$-fiber nociceptive pulpal afferents. ${ }^{27}$ Hence, the direct activation of TRPA1 ion channels and C-fiber firing could explain the dull and annoying pain caused by bleaching agents. ${ }^{28}$ As hypothesized by Markowitz, ${ }^{29}$ the release of ATP and prostaglandins that accompany pulp tissue damage would have an excitatory effect on pulpal nociceptors and consequently lead to peripheral sensitization through neurogenic inflammation caused by increased levels of vasoactive neuropeptides such as SP and CGRP in pulpal tissue.

\section{Dental nociceptor contribution to peripheral and central sensitization}

Upon noxious thermal, mechanical and chemical stimulation of the nerve terminals within the pulpodentin complex, the activation of peptidergic fibers causes the local release of vasoactive neuropeptides such as SP and CGRP. These neuropeptides are potent vasodilators, and they produce plasma extravasation and cell release of chemical mediators that act on certain receptors and ion channels of the nociceptive nerve endings. By virtue of their action nerve endings, activation thresholds are lowered and may show an enhanced response to subsequent noxious stimulation. ${ }^{30,31}$ Consequently, nerve endings may start responding to innocuous stimuli. By their effect on vascular permeability, pulpal blood pressure, mast cells and other immunocompetent cells, the spreading of SP and CGRP released by peptidergic fibers contributes to the genesis and maintenance of local neurogenic inflammation. Hence, peripheral sensitization then leads to hyperalgesia, allodynia and spreading pain. The sustained barrage of peripheral nerve inputs mediated by neurogenic inflammation to the trigeminal brainstem nuclear complex may lead to prolonged neuroplastic alterations in nociceptive pathways and thus central sensitization. Dental primary afferent nociceptive fibers can undergo phenotypic changes and start responding to non-painful stimuli. ${ }^{30}$ The mechanisms of trigeminal central sensitization are complex and underlie the increased excitability of nociceptive pathways secondary to inflammation and peripheral tissue injury. ${ }^{32}$ Interestingly, the upregulation of nerve growth factors (NGFs) in inflamed tissue can sensitize TRPV1 ion channels and thus participate in peripheral sensitization and inflammatory heat pain hypersensitivity. ${ }^{16}$ Both TRPV1 and TRPA1 may also play an integral role in pain and neurogenic inflammation via sensory nerve activation at both a central and a peripheral level. ${ }^{15}$

\section{Discussion}

The most widely endorsed explanation for DH (localized, sharp, brief, acute pain) is the involvement of the A $\delta$-fibers by the activation of mechanosensitive nociceptors via dentinal fluid movement in the tubules (hydrodynamic theory). This dental allodynia (diffuse, dull, deep, sustained pain) induced by an ambient thermal differential that goes from cold to warm but not the reverse would involve sensitized C-fibers expressing mechano- and heat-sensitive nociceptors as well as central sensitization processes. After repeated exposure to a temperature gradient, phenotypic changes are taking place in pulpal nerve endings of dental afferents in selected teeth. Peptidergic fibers become responsive to innocuous environmental stimuli, which result in a prime state of "silent" or "low-grade" neurogenic inflammation induced by the release of vasoactive neuropeptides. Teeth are asymptomatic but have a hyperresponsiveness to electrical testing. This suggests a lowering of the pain threshold of C-fibers at the thermal level. With the installment of peripheral sensitization, the activation threshold of TRPV1 ion channels is lowered, and C-fibers become responsive to usually innocuous temperature gradient (from cold to warm). Hence, recurrent activation of C-fibers can set off the hemodynamic alterations leading to a transient state of pulp inflammation. Moreover, the activation of heat-sensitive TRPV1 channels co-localized on nonneuronal cells can contribute to the release of inflammatory mediators ${ }^{15}$ and play an important role in thermal hyperalgesia associated with the mediation of pulpal inflammation. ${ }^{14}$ Co-expression of TRPA1 and TRPV1 could also mediate the function of polymodal nociceptors and play an integral role in pain and neurogenic inflammation via sensory nerve activation. ${ }^{15}$ Hypotheses of possible mechanisms underlying dental thermal allodynia are depicted in Figure 1.

Changes in PBF and the hydrodynamic force with resulting shear stresses associated with dentinal fluid movements are among potential mechanisms that could underlie the activation of sensitized nerve terminals. Fluid flow rate in a dentinal tubule can trigger nerve impulses and generate a sensory response in healthy teeth. It has been shown by Lin et $\mathrm{al}^{9}$ that compared to pure warming there is an increased 


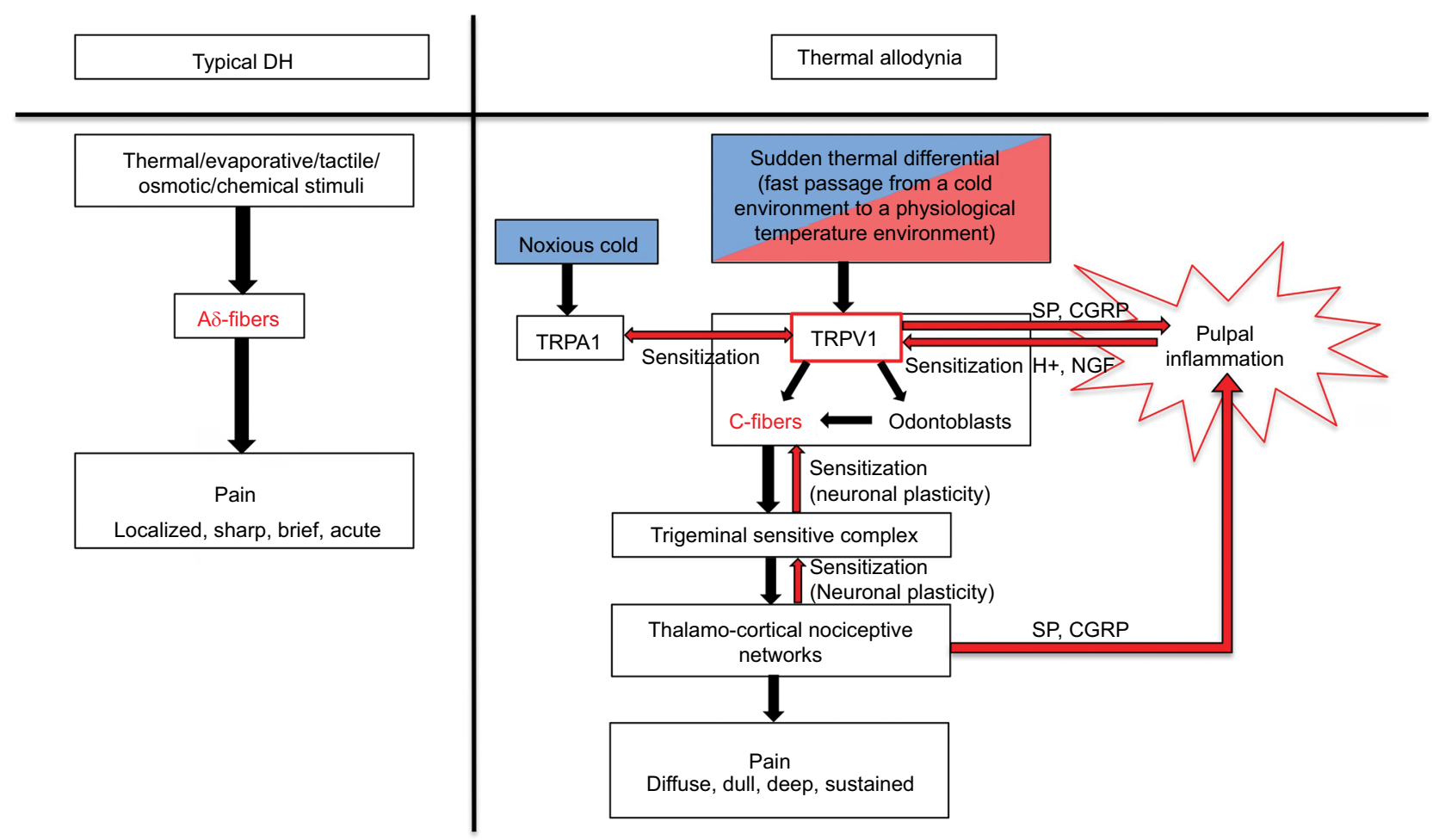

Figure I Hypothesis of possible mechanisms underlying dental thermal differential allodynia. Abbreviations: $\mathrm{DH}$, dentin hypersensitivity; NGF, nerve growth factor.

fluid flow velocity and neural discharge frequency in a tooth previously exposed to cold.. They estimated that the outward fluid flow velocity was $140 \%$ larger than that induced by pure cooling when heating preceded cooling. This clearly suggests that a tooth with sensitized nociceptors could have an enhanced response to a temperature differential that is normally not painful.

\section{Conclusion}

This review focused on patients presenting an abnormal thermal differential allodynia inducing delayed dental pain when they changed from a cold environment to a warm environment. Recognizing dental pain induced by thermal differential as being different from classical DH is important since it is likely that different pathophysiological processes underlie both conditions. Ignoring that temperature gradient may set off episodes of dentoalveolar pain that can lead to misdiagnosis and unnecessary treatment. As more is understood about this singular condition, better patient care as well as better preventive and treatment modalities will emerge. Novel approaches using a TRP antagonist might be a potential avenue for treating thermal allodynia mediated by intradental nerve sensitization. ${ }^{33,34}$

\section{Disclosure}

The authors report no conflicts of interest in this work.

\section{References}

1. Canadian Advisory Board on Dentin Hypersensitivity. Consensusbased recommendations for the diagnosis and management of dentin hypersensitivity. J Can Dent Assoc. 2003;69(4):221-226.

2. Holland GR, Narhi MN, Addy M, Gangarosa L, Orchardson R. Guidelines for the design and conduct of clinical trials on dentine hypersensitivity. J Clin Periodontol. 1997;24(11):808-813.

3. Paik S, Park K, Lee S, et al. Light and electron microscopic analysis of the somata and parent axons innervating the rat upper molar and lower incisor pulp. Neuroscience. 2009;162(4):1279-1286.

4. Djouhri L, Lawson SN. Abeta-fiber nociceptive primary afferent neurons: a review of incidence and properties in relation to other afferent A-fiber neurons in mammals. Brain Res Brain Res Rev. 2004;46(2): 131-145.

5. Lin M, Xu F, Lu TJ, Bai BF. A review of heat transfer in human tooth experimental characterization and mathematical modeling. Dent Mater. 2010;26(6):501-513.

6. Linsuwanont P, Palamara JEA, Messer HH. An investigation of thermal stimulation in intact teeth. Arch Oral Biol. 2007;52(3):218-227.

7. Linsuwanont P, Versluis A, Palamara JE, Messer HH. Thermal stimulation causes tooth deformation: a possible alternative to the hydrodynamic theory? Arch Oral Biol. 2008;53(3):261-272.

8. West NX, Lussi A, Seong J, Hellwig E. Dentin hypersensitivity: pain mechanisms and aetiology of exposed cervical dentin. Clin Oral Investig. 2013;17(Suppl 1):S9-S19.

9. Lin M, Liu S, Niu L, Xu F, Lu TJ. Analysis of thermal-induced dentinal fluid flow and its implications in dental thermal pain. Arch Oral Biol. 2011;56(9):846-854. 
10. Brännström M, Johnson G. Movements of the dentine and pulp liquids on application of thermal stimuli. An in vitro study. Acta Odontol Scand. 1970;28(1):59-70.

11. Lindén L, Brännström M. Fluid movements in dentine and pulp. An in vitro study of flow produced by chemical solutions on exposed dentine. Odontol Revy. 1967;18(3):227-236.

12. Chung G, Jung SJ, Oh SB. Cellular and molecular mechanisms of dental nociception. J Dent Res. 2013;92(11):948-955.

13. Kim HY, Chung G, Jo HJ, et al. Characterization of dental nociceptive neurons. J Dent Res. 2011;90(6):771-776.

14. El Karim IA, Linden GJ, Curtis TM, et al. Human odontoblasts express functional thermo-sensitive TRP channels: implications for dentin sensitivity. Pain. 2011;152(10):2211-2223.

15. Ji R-R, Samad TA, Jin S-X, Schmoll R, Woolf CJ. p38 MAPK activation by NGF in primary sensory neurons after inflammation increases TRPV1 levels and maintains heat hyperalgesia. Neuron. 2002;36(1):57-68.

16. Tominaga M, Caterina MJ. Thermosensation and pain. J Neurobiol. 2004;61(1):3-12.

17. Magloire H, Maurin JC, Couble ML, et al. Topical review. Dental pain and odontoblasts: facts and hypotheses. J Orofac Pain. 2010;24(4): 335-349.

18. Egbuniwe O, Grover S, Duggal AK, et al. TRPA1 and TRPV4 activation in human odontoblasts stimulates ATP release. J Dent Res. 2014;93(9):911-917.

19. Maurin J-C, Couble M-L, Thivichon-Prince B, Magloire H. L'odontoblaste: Un acteur incontournable de la perception de la douleur dentinaire [Odontoblast: a key cell involved in the perception of dentinal pain]. Med Sci (Paris). 2013;29(3):293-299. French.

20. Shibukawa Y, Sato M, Kimura M, et al. Odontoblasts as sensory receptors: transient receptor potential channels, pannexin-1, and ionotropic ATP receptors mediate intercellular odontoblast-neuron signal transduction. Pflugers Arch. 2015;467(4):843-863.

21. El Karim I, McCrudden M, Linden GJ, et al. TNF- $\alpha$-induced p38MAPK activation regulates TRPA 1 and TRPV4 activity in odontoblast-like cells. Am J Pathol. 2015;185(11):2994-3002.
22. Lehmuskallio E, Hassi J, Kettunen P. The skin in the cold. Int J Circumpolar Health. 2002;61(3):277-286.

23. Bakst R, Merola JF, Franks AG, Sanchez M. Raynaud's phenomenon: pathogenesis and management. J Am Acad Dermatol. 2008; 59(4):633-653.

24. Baldissara P, Catapano S, Scotti R. Clinical and histological evaluation of thermal injury thresholds in human teeth: a preliminary study. J Oral Rehabil. 1997;24(11):791-801.

25. Haywood VB. Treating sensitivity during tooth whitening. Compend Contin Educ Dent. 2005;26(9 Suppl 3):11-20.

26. Costa CA, Riehl H, Kina JF, Sacono NT, Hebling J. Human pulp responses to in-office tooth bleaching. Oral Surg Oral Med Oral Pathol Oral Radiol Endod. 2010;109(4):e59-e64.

27. Kobayashi K, Fukuoka T, Obata K, et al. Distinct expression of TRPM8, TRPA1, and TRPV1 mRNAs in rat primary afferent neurons with adelta/ c-fibers and colocalization with trk receptors. J Comp Neurol. 2005; 493(4):596-606

28. Thiesen $\mathrm{CH}$, Rodrigues Filho R, Prates LHM, Sartori N. The influence of desensitizing dentifrices on pain induced by in-office bleaching. Braz Oral Res. 2013;27(6):517-523.

29. Markowitz K. Pretty painful: why does tooth bleaching hurt? Med Hypotheses. 2010;74(5):835-840.

30. Dallel R, Villanueva L, Woda A, Voisin D. Neurobiologie de la douleur trigéminale [Neurobiology of trigeminal pain]. Med Sci (Paris). 2003;19(5):567-574. French.

31. Sessle BJ. Peripheral and central mechanisms of orofacial inflammatory pain. Int Rev Neurobiol. 2011;97:179-206.

32. Xie YF, Zhang S, Chiang CY, Hu JW, Dostrovsky JO, Sessle BJ. Involvement of glia in central sensitization in trigeminal subnucleus caudalis (medullary dorsal horn). Brain Behav Immun. 2007;21(5):634-641.

33. Derry S, Sven-Rice A, Cole P, Tan T, Moore RA. Topical capsaicin (high concentration) for chronic neuropathic pain in adults. Cochrane Database Syst Rev. 2013;2:CD007393.

34. Gualdani R, Ceruti S, Magni G, et al. Lipoic-based TRPA1/TRPV1 antagonist to treat orofacial pain. ACS Chem Neurosci. 2015;6(3):380-385.
Journal of Pain Research

\section{Publish your work in this journal}

The Journal of Pain Research is an international, peer reviewed, open access, online journal that welcomes laboratory and clinical findings in the fields of pain research and the prevention and management of pain. Original research, reviews, symposium reports, hypothesis formation and commentaries are all considered for publication.

\section{Dovepress}

The manuscript management system is completely online and includes a very quick and fair peer-review system, which is all easy to use. Visit http://www.dovepress.com/testimonials.php to read real quotes from published authors. 